\title{
Haplotipos en los genes de las metaloproteinasas 7, 8, 12 y 13 asociados con cáncer colorrectal en población mexicana
}

José M. Moreno-Ortiz1, Melva Gutiérrez-Angulo², Ruth Ramírez-Ramírez³, Anahí González-Mercado', Alexis S. Suárez-Villanueva ${ }^{3,4}$, Miriam Partida-Pérez ${ }^{5}$, Víctor Maciel-Gutiérrez ${ }^{6}$ y

Ma. de la Luz Ayala-Madrigal *

${ }^{1}$ Departamento de Biología Molecular y Genómica, Instituto de Genética Humana Dr. Enrique Corona Rivera, Centro Universitario de Ciencias de la Salud, Universidad de Guadalajara; ${ }^{2}$ Departamento de Ciencias de la Salud, Centro Universitario Los Altos, Universidad de Guadalajara; ${ }^{3}$ Departamento de Biología Celular y Molecular, Centro Universitario de Ciencias Biológicas y Agropecuarias, Universidad de Guadalajara; ${ }^{4}$ Escuela de Medicina, Campus Zapopan, Universidad del Valle de México, Zapopan; ${ }^{5}$ Departamento de Ciencias Médicas, Centro Universitario de la Costa, Universidad de Guadalajara; ${ }^{6}$ Servicio de Colon y Recto, Hospital Civil Dr. Juan I. Menchaca, Guadalajara. Jalisco, Mexico

\section{Resumen}

Antecedentes: Las metaloproteinasas (MMP) se involucran en invasión y progresión tumoral en cáncer colorrectal (CCR). Las variantes rs11568818, rs11225395, rs2276109 y rs2252070 se han asociado con esta neoplasia. Objetivo: Evaluar haplotipos de las MMP 7, 8, 12, y 13 y su asociación con CCR. Material y métodos: Se genotipificaron 104 pacientes y 112 individuos sanos mediante reacción en cadena de la polimerasa con análisis del polimorfismo de los fragmentos de restricción (PCR-RFLP). Para el análisis de asociación fueron calculados valores de odds ratio e intervalo de confianza. El análisis de haplotipos y desequilibrio de ligamiento $(L D)$ se realizó con el software Arlequin v3.5. Resultados: Se presentó $L D$ entre rs2276109 y rs2252070. Los haplotipos rs11568818(A)-rs11225395(T)-rs2276109(A)-rs2252070(A) y rs11568818(A)rs11225395(C)-rs2276109(A)-rs2252070(G) se asociaron con riesgo de CCR y los haplotipos rs11568818(G)-rs11225395(C)rs2276109(A)-rs2252070(A) y rs11568818(A)-rs11225395(T)-rs2276109(A)-rs2252070(G) con protección. Conclusión: Las variantes rs2276109 y rs2252070 mostraron ligamiento génico. Dos haplotipos fueron asociados con el desarrollo de CCR (ATAA y ACAG) y dos fueron asociados con protección (GCAA y ATAG). Este estudio representa el primer reporte de frecuencias de las variantes rs11225395 y rs2276109 en población mexicana.

PALABRAS CLAVE: Cáncer colorrectal. Haplotipos. Metaloproteinasas. Población mexicana.

\section{Matrix metalloproteinases 7, 8, 12, 13 gene haplotypes associated with colorectal cancer in a Mexican population}

\section{Abstract}

Background: Matrix metalloproteinases (MMPS) are involved in tumor invasion and progression in colorectal cancer (CRC). Variants rs11568818, rs11225395, rs2276109 and rs2252070 have been associated with this neoplasm. Objective: To evaluate MMPs 7, 8, 12, and 13 haplotypes and their association with CRC. Material and methods: One-hundred and four patients and 112 healthy individuals were genotyped by polymerase chain reaction-restriction fragment length polymorphism analysis (PCR-RFLP). For the association analysis, odd ratio and confidence interval values were calculated. Haplotype and linkage disequilibrium (LD) analysis was performed with Arlequin software, v3.5. Results: $L D$ was present between rs2276109 and rs2252070. Haplotypes rs11568818(A)-rs11225395(T)-rs2276109(A)-rs2252070(A) and rs11568818(A)-rs11225395(C)-

Correspondencia:

${ }^{*}$ Ma. de la Luz Ayala-Madrigal

E-mail: luz.ayala@academicos.udg.mx
Gac Med Mex. 2021;157:548-553

Disponible en PubMed

www.gacetamedicademexico.com 0016-3813/@ 2021 Academia Nacional de Medicina de México, A.C. Publicado por Permanyer. Este es un artículo open access bajo la licencia CC BY-NC-ND (http://creativecommons.org/licenses/by-nc-nd/4.0/). 
rs2276109(A)-rs2252070(G) were associated with CRC risk, and the haplotypes rs11568818(G)-rs11225395(C)-rs2276109(A)rs2252070(A) and rs11568818(A)-rs11225395(T)-rs2276109(A)-rs2252070(G) with protection. Conclusion: Variants rs2276109 and rs2252070 showed linkage genic. Two haplotypes were found associated with the development CRC (ATAA and ACAG) and two were associated with protection (GCAA and ATAG). This study represents the first report on variants rs 11225395 and rs2276109 frecuency in a Mexican population.

KEY WORDS: Colorectal cancer. Haplotypes. Matrix metalloproteinases. Mexican population.

\section{Introducción}

El cáncer colorrectal (CCR) es uno de los cánceres con mayor prevalencia en el mundo, su incidencia y mortalidad varían marcadamente entre los países ${ }^{1}$. En población mexicana, el CCR es la tercera causa más común de mortalidad ${ }^{2}$. La carcinogénesis en CCR es un proceso complejo donde células normales sufren diferentes alteraciones genéticas y epigenéticas, transformándose en células malignas ${ }^{3}$. En este proceso, las metaloproteinasas (MMP) participan en la remodelación y degradación de proteínas de la matriz extracelular (MEC) y por ello están involucradas en la progresión e invasión tumoral al catalizar la proliferación, migración y diferenciación celular ${ }^{4,5}$. Los genes de las metaloproteasas se encuentran localizados muy próximos, entre chr11:102530930 -102955810 (GRCh38.p13). Variantes en el promotor de los genes de MMP han sido asociadas con $\mathrm{CCR}^{6-8}$. MMP7 rs11568818 (-181A>G), MMP8 rs11225395 (-799C>T), MMP12 rs2276109 (-82A>G), y MMP13 rs2252070 $(-77 \mathrm{~A}>\mathrm{G})$ han sido implicados en varios tipos de cáncer. Por lo que el propósito del presente estudio fue inferir haplotipos entre estas variantes y su posible asociación con CCR en pacientes mexicanos.

\section{Material y métodos}

\section{Sujetos}

En este estudio se incluyeron 104 pacientes con diagnóstico histopatológico de adenocarcinoma colorrectal que no hubieran recibido tratamiento con radioterapia ni quimioterapia. El grupo de referencia estuvo formado por 112 donadores de sangre periférica sanos. Todas las muestras fueron captadas entre diciembre de 2008 y diciembre de 2013, así como también se obtuvo un consentimiento informado de los participantes del Hospital Civil de Guadalajara Dr. Juan I. Menchaca. Fueron aplicados cuestionarios para evaluar el estilo de vida de los pacientes, además de que se revisaron los expedientes médicos para obtener las características clínico-patológicas. El estudio cumple los criterios de la Declaración de Helsinki, y los comités de ética e investigación del Centro Universitario de Ciencias de la Salud de la Universidad de Guadalajara y del Hospital Civil Dr. Juan I. Menchaca de Guadalajara, Jal., México, aprobaron el estudio (número de registro $\mathrm{Cl}-14409$ y 935/09, respectivamente).

\section{Genotipificación}

EI DNA fue extraído a partir de muestras de sangre periférica tanto de pacientes como de individuos del grupo de población general, usando una modificación del método CTAB-DTAB ${ }^{9}$. Las variantes fueron genotipificadas por reacción en cadena de la polimerasa con análisis del polimorfismo de los fragmentos de restricción (PCR-RFLP). Las condiciones de genotipificación y digestión para rs11568818 (MMP7) y rs2252070 (MMP13) fueron descritas por nuestro grupo de trabajo ${ }^{10}$. Se diseñaron los siguientes iniciadores para identificar la variante rs11225395 (MMP8): 5'-AGCTGCTGCTCCACTATG-3' y 5'-TCTGGAGG ATGTGGTTTG-3'. Para identificar rs2276109 (MMP12) fueron usados iniciadores reportados previamente ${ }^{11}$. Las reacciones de PCR se realizaron en un volumen de $25 \mu$ l conteniendo $100 \mathrm{ng}$ de DNA. La PCR para rs11225395 (MMP8) consistió en 26 ciclos y la desnaturalización se realizó a una temperatura de $94^{\circ} \mathrm{C}$, la alineación a $63.1^{\circ} \mathrm{C}$ y la elongación a $72{ }^{\circ} \mathrm{C}$ por 30 s. Condiciones similares de PCR fueron usadas para la variante rs2276109 (MMP12), con la excepción de que la alineación fue hecha a $55^{\circ} \mathrm{C}$ por 40 ciclos. Los productos de PCR fueron sometidos a digestión con las enzimas Sfcl y HpyCH4II para rs11225395 (MMP8) y rs2276109 (MMP12), respectivamente. Se usaron cuatro unidades de la enzima y $10 \mu \mathrm{l}$ del producto de PCR. Los fragmentos observados para rs 11225395 (MMP8) correspondieron a 399 pb para el alelo silvestre (C) y 336 pb y 62 pb para el alelo polimórfico T. Para rs2276109 (MMP12), los fragmentos observados fueron 163 pb y 22 pb para el alelo silvestre $(A)$ 
y $101 \mathrm{pb}, 62 \mathrm{bp}$, y $22 \mathrm{pb}$ para el alelo polimórfico (G). Como control de calidad de la genotipificación, esta fue repetida aleatoriamente en el $20 \%$ de las muestras. Para el control positivo de la digestión, las enzimas de restricción fueron usadas con el vector bacteriófago Lambda. Los fragmentos fueron visualizados en geles de poliacrilamida al $6 \%$ teñidos con nitrato de plata.

\section{Análisis estadístico}

Las frecuencias alélicas y genotípicas fueron estimadas por conteo directo. Se determinó el equilibrio de Hardy-Weinberg (EHW) usando chi cuadrada. Para medir la asociación entre las variantes y el CCR, fueron calculados las odds ratios (OR) y un intervalo de confianza del 95\% (IC 95\%). Se utilizó el software Arlequin $\mathrm{v} 3.5^{12}$ para realizar el análisis haplotípico y el desequilibrio de ligamiento (DL) fue medido usando los coeficientes $r^{2}$ y D'. Valores de $r^{2}>0.33$ indicaron un fuerte $\mathrm{DL}$, y $\mathrm{D}^{\prime}=1$ indicó completo $\mathrm{DL}^{13,14}$. Para todos los análisis estadísticos se consideró como significativo un valor de $p<0.05$.

\section{Resultados}

Las características de los 104 pacientes con CCR se muestran en la tabla 1 . En estos pacientes, la localización del tumor fue principalmente en el colon (55\%). El grupo de referencia estuvo caracterizado por individuos mayores de 18 años, de los cuales el $58 \%$ fueron varones. La distribución de los alelos y los genotipos para las variantes rs11225395 (MMP8) y rs2276109 (MMP12) estuvieron en EHW ( $p>0.05)$, y no fue encontrada asociación significativa. El análisis de SNV (single nucleotide variant), incluyendo los modelos genéticos se muestran en la tabla 2. Las frecuencias para rs11568818 (MMP7) y rs2252070 (MMP13) fueron descritas en trabajos previos ${ }^{10}$.

El análisis de DL fue realizado considerando los resultados de las frecuencias genotípicas reportadas por Moreno-Ortiz, et al. (2014) para rs11568818 (MMP7) y rs2252070 (MMP13). Las frecuencias genotípicas de las variantes rs11225395 (MMP8) y rs2276109 (MMP12) mostraron desequilibrio para rs2276109 (MMP12) y rs2252070 (MMP13) con D' = 1 para el grupo de referencia y $D^{\prime}=0.74$ para los pacientes, y están reportadas en la tabla 3.

La inferencia de haplotipos para todas las SNV llevó a la identificación de 11 haplotipos para el grupo de referencia y 12 para el grupo de pacientes con CCR.
Tabla 1. Características de los pacientes con cáncer colorrectal (CCR)

\begin{tabular}{l|c|}
\hline Características & $\begin{array}{c}\text { Pacientes con CCR } \\
(\mathbf{n}=104)\end{array}$ \\
\hline Edad (años) & $59 \pm 14$ \\
\hline Peso (kg) & $68 \pm 15$ \\
Altura (m) & $1.7 \pm 10$ \\
Sexo & \\
Masculino & $53 \%$ \\
Femenino & $47 \%$ \\
Presencia de DM2 & \\
Sí & \\
No & $80 \%$ \\
ND & $16 \%$ \\
Sobrepeso y/o obesidad & $4 \%$ \\
Sí & \\
No & $24 \%$ \\
ND & $52 \%$ \\
Consumo de alcohol & $24 \%$ \\
Sí & \\
No & \\
ND & $58 \%$ \\
Consumo de tabaco & $34 \%$ \\
Sí & $8 \%$ \\
No & \\
ND & \\
Actividad física & $57 \%$ \\
Sí & $39 \%$ \\
No & $4 \%$ \\
\hline
\end{tabular}

DM2: diabetes mellitus 2; ND: datos no disponibles.

Sin embargo, únicamente son presentados en la tabla 4 aquellos con una frecuencia mayor al $10 \%$ en cada uno de los grupos. Los haplotipos rs11568818(A)rs11225395(T)-rs2276109(A)-rs2252070(A) y rs115 68818(A)-rs11225395(C)-rs2276109(A)-rs2252070(G) mostraron un incremento del riesgo de CCR, y los haplotipos rs11568818(G)-rs11225395(C)-rs22761 09(A)-rs2252070(A) y rs11568818(A)-rs112253 95(T)-rs2276109(A)-rs2252070(G) fueron asociados con protección contra el CRC.

\section{Discusión}

En diferentes tipos de cáncer, la expression y la activación de MMP son una marca importante en la progresión tumoral, incluyendo angiogénesis, invasión y metástasis. Niveles elevados de MMP están también relacionados con sobrevivencia ${ }^{5}$. Variantes específicas en los genes de MMP y sus haplotipos 
Tabla 2. Comparación de las frecuencias genotípicas de las variantes rs11225395 y rs2276109 entre cáncer colorrectal (CCR) y el grupo de referencia (sano)

\begin{tabular}{|c|c|c|c|c|c|}
\hline \multirow[t]{2}{*}{ Gen/variante } & \multirow[t]{2}{*}{ Genotipo/alelo } & $\begin{array}{l}\text { Grupo de referencia (sano) } \\
\text { n (\%) }\end{array}$ & $\begin{array}{l}\text { Pacientes con CCR } \\
\mathrm{n}(\%)\end{array}$ & \multirow[t]{2}{*}{$\mathrm{p}^{*}$} & \multirow[t]{2}{*}{ OR } \\
\hline & & $\mathrm{n}=112$ & $\mathrm{n}=102$ & & \\
\hline \multirow[t]{8}{*}{ MMP8/rs11225395 } & CC & $40(36)$ & $32(31)$ & & 1.0 (referencia) \\
\hline & СT & $53(47)$ & $53(52)$ & 0.46 & $1.25(0.68-2.27)$ \\
\hline & TT & $19(17)$ & $17(17)$ & 0.78 & $1.11(0.50-2-49)$ \\
\hline & C & $133(59)$ & $117(57)$ & & 1.0 (referencia) \\
\hline & T & $91(41)$ & $87(43)$ & 0.67 & $1.08(0.73-1.59)$ \\
\hline & $\mathrm{CT}+\mathrm{TT}$ & $93(83)$ & $85(83)$ & & 1 (referencia) \\
\hline & CC & $19(17)$ & $17(17)$ & 0.95 & $0.97(0.47-2.00)$ \\
\hline & & $n=102$ & $n=104$ & & \\
\hline \multirow[t]{7}{*}{ MMP12/rs2276109 } & AA & $89(87)$ & $89(86)$ & & 1.0 (referencia) \\
\hline & $A G$ & $11(11)$ & $14(13)$ & 0.57 & $1.27(0.54-2.95)$ \\
\hline & $G G$ & 2 (2) & $1(1)$ & 0.57 & $0.50(0.04-5.61)$ \\
\hline & A & 189 (93) & $192(92)$ & & 1.0 (referencia) \\
\hline & G & $15(7)$ & $16(8)$ & 0.89 & $1.05(0.50-2.18)$ \\
\hline & $A G+G G$ & $100(98)$ & $103(99)$ & & 1 (referencia) \\
\hline & AA & 2 (2) & $1(1)$ & 0.55 & $0.48(0.04-5.43)$ \\
\hline
\end{tabular}

podrían tener un impacto en su proteína. En el presente estudio, las variantes rs2276109 (MMP12) y rs2252070 (MMP13) mostraron desequilibrio, esto debido principalmente a la distancia que hay entre ellas por su posición en el cromosoma. Mientras que la variante rs2276109 (MMP12) está localizada en chr11:102875061, la variante rs2252070 (MMP13) está localizada chr11:102955810; esto es, que entre ellas hay una distancia de 80,749 pb. En el cromosoma, son los genes más cercanos uno del otro en comparación con las otras MMP analizadas. El análisis de DL fue llevado a cabo para controles y pacientes con CCR independientemente para determinar el riesgo conferido por estos marcadores genéticos. La combinación de estas SNV estuvieron en perfecto DL ( $D^{\prime}=1$ ), demostrando su fuerte asociación.

No ha habido estudios que hayan analizados haplotipos con las variantes presentadas en este estudio. El principal haplotipo identificado como un factor de riesgo para CCR fueron MMP7 rs11568818(A)-MMP8 rs11225395(T)-MMP12 rs2276109(A)-MMP13 rs22520 70(A) (OR: 5.6; IC 95\%: 2.4-12.8; $p=0.001$ ) y MMP7
Tabla 3. Desequilibrio de ligamiento entre las variantes analizadas

\begin{tabular}{|c|c|c|c|c|}
\hline \multirow[t]{2}{*}{ Gen/variante } & \multicolumn{2}{|c|}{$\begin{array}{l}\text { Grupo de } \\
\text { referencia (sano) }\end{array}$} & \multicolumn{2}{|c|}{ Pacientes con CCF } \\
\hline & $D^{\prime}$ & $r^{2}$ & $D^{\prime}$ & $r^{2}$ \\
\hline \multirow{4}{*}{$\begin{array}{l}\text { MMP7/rs11568818 } \\
\text { MMP8/rs11225395 } \\
\text { MMP12/rs2276109 } \\
\text { MMP13/rs2252070 }\end{array}$} & & & & \\
\hline & 0.21 & 0.01 & 0.29 & 0.05 \\
\hline & 0.82 & 0.07 & 0.32 & 0.02 \\
\hline & 0.35 & 0.07 & 0.59 & 0.23 \\
\hline \multicolumn{5}{|l|}{ MMP8/rs11225395 } \\
\hline MMP12/rs2276109 & 0.74 & 0.02 & 0.84 & 0.34 \\
\hline MMP13/rs2252070 & 0.51 & 0.10 & 0.27 & 0.03 \\
\hline \multicolumn{5}{|l|}{ MMP12/rs2276109 } \\
\hline MMP13/rs2252070 & 1.00 & 0.20 & 0.74 & 0.15 \\
\hline
\end{tabular}

CCR: cáncer colorrectal.

rs11568818(A)-MMP8 rs11225395(C)-MMP12 rs2276 109(A)-MMP13 rs2252070(G) (OR: 3.1; IC 95\%: 1.27.9; $p=0.001)$. En estos haplotipos, los alelos que los conforman han sido asociados con incremento 0 reducción del riesgo e incluso protección contra diferentes tipos de cáncer como $\mathrm{CCR}^{10,15}$, cáncer de hígado ${ }^{15,16}$, cáncer de mama, cáncer de pulmón, 
Tabla 4. Frecuencias haplotípicas y su asociación con CCR

\begin{tabular}{|c|c|c|c|c|c|c|c|c|}
\hline \# & $\begin{array}{c}\text { MMP7/ } \\
\text { rs11568818 }\end{array}$ & $\begin{array}{c}\text { MMP8/ } \\
\text { rs11225395 }\end{array}$ & $\begin{array}{l}\text { MMP12/ } \\
\text { rs2276109 }\end{array}$ & $\begin{array}{l}\text { MMP13/ } \\
\text { rs2252070 }\end{array}$ & $\begin{array}{l}\text { Grupo de } \\
\text { referencia (sano) }\end{array}$ & Pacientes con CCR & OR (IC 95\%) & $p$ \\
\hline 1 & A & C & $A$ & $A$ & 0.46 & 0.72 & 1.0 (referencia) & \\
\hline 2 & $A$ & $\mathrm{~T}$ & $A$ & A & 0.44 & 0.39 & $5.6(2.4-12.8)$ & $p=0.00$ \\
\hline 3 & $G$ & C & A & A & 0.36 & 0.10 & $0.2(0.1-0.4)$ & $p=0.00$ \\
\hline 4 & A & C & A & G & 0.19 & 0.11 & $3.1(1.2-7.9)$ & $p=0.01$ \\
\hline 5 & $A$ & $\mathrm{~T}$ & $A$ & $\mathrm{G}$ & 0.18 & 0.90 & $0.3(0.1-0.8)$ & $p=0.01$ \\
\hline 6 & $G$ & $\mathrm{~T}$ & A & G & 0.14 & 0.18 & $1.7(0.6-5.2)$ & $p=0.30$ \\
\hline 7 & $G$ & $\mathrm{~T}$ & $A$ & $A$ & 0.09 & 0.19 & $0.6(0.1-2.9)$ & $p=0.50$ \\
\hline
\end{tabular}

CCR: cáncer colorrectal; OR: odds ratio; IC 95\%: intervalo de confianza del $95 \%$.

carcinoma hepatocelular, adenocarcinoma esofageal y cáncer gástrico ${ }^{15}$. Específicamente, el genotipo homocigoto AA y el heterocigoto AG para MMP7 (rs11568818) fueron asociados con riesgo para CCR. Por el contrario, los individuos con genotipos AA de MMP13 (rs2252070) presentaron tres veces más riesgo de desarrollar $\mathrm{CCR}^{10}$. Para la variante localizada en MMP13, esta se encuentra en una secuencia consenso para el factor de transcripción PEA3, donde la presencia del alelo A incrementa doblemente la actividad del promotor ${ }^{17}$. Esta variante está asociada con un riesgo altamente agresivo de cáncer oral y el alelo A parece ser un factor pronóstico para la progresión tumoral ${ }^{18}$. Respecto a MMP12 (rs2276109), el alelo A ha sido encontrado asociado a una elevada actividad del promotor ${ }^{8,15}$, desde que esta variante está localizada en el sitio de unión para factor de transcripción AP1, demostrándose una mayor afininidad ${ }^{8,19}$. Para la variante MMP8 (rs11225395), el alelo T fue asociado con el incremento de riesgo de cáncer de hígado en una población americana ${ }^{16}$.

Los haplotipos MMP7 rs11568818(G)-MMP8 rs11225395(C)-MMP12 rs2276109(A)-MMP13 rs2252070(A) (OR: 0.2; IC 95\%: 0.1-0.4; $p=0.01$ ) y MMP7 rs11568818(A)-MMP8 rs11225395(T)-MMP12 rs2276109(A)-MMP13 rs2252070(G) (OR: 0.3; IC 95\%: $0.1-0.8 ; p=0.01$ ) parecen ser factores protectores contra el CCR. Algunos de los alelos que conforman estos haplotipos, además de ser asociados con un riesgo también han sido identificados por tener efectos protectores contra el CCR. El alelo $\mathrm{T}$ de la variante rs11225395 (MMP8) ha sido relacionado con baja susceptibilidad para desarrollar metástasis y además ha indicado un mejor pronóstico de superviviencia en pacientes con cáncer de mama. De igual manera, en cáncer oral, varios reportes han mostrado una baja susceptibilidad para cáncer oral ${ }^{20}$. Adicionalmente, no ha habido relaciones significativas en niños con leucemia en población taiwanesa ${ }^{21}$.

En este sentido, no ha habido predominio de algún alelo específico. Sin embargo, es interesante mencionar que en la variante rs11225395 (MMP8) el alelo T ha sido asociado con protección en diferentes tipos de cáncer $^{20,21}$. En este estudio, la comparación de rs11225395 (MMP8) entre el grupo de referencia y el grupo de pacientes con CCR no mostró diferencias significativas $(p>0.05)$. Sin embargo, este reporte representa el primer estudio de asociación de la variante rs11225395 con CCR en población mexicana. Respecto a rs2276109 (MMP12), es importante mencionar que el alelo $\mathrm{G}$ tiene las frecuencias más bajas comparadas con las otras variantes analizadas (MAF $=0.051000$ Genome Project) y que la ausencia del alelo $G$ podría posiblemente jugar un rol en la disminución del riesgo de CCR.

Es importante mencionar que en el haplotipo MMP7 rs11568818(A)-MMP8 rs11225395(T)-MMP12 rs2276109(A)-MMP13 rs2252070(A), comparado con otro analizado: MMP7-181A-MMP1-1607dupG-MMP31171A-MMP12-82A, el alelo A predominó para las variantes en MMP7 y MMP12. Además, el haplotipo analizado fue más frecuente en pacientes con CCR que en controles sanos ${ }^{8}$. Luego entonces, podría ser que existe una fuerza haplotípica mayor derivada de la presencia de un alelo específico y no de la suma de todos los alelos. Por otro lado, en el análisis de haplotipos de tres SNV (MMP8 rs2509013 C>T/MMP8 rs11225395 G>A/MMP27 rs3809017 T>C) ${ }^{22}$, la mayoría de los haplotipos que tuvieron $T$ fueron asociados con una disminución en la recurrencia de embarazos, por lo que la influencia de un único alelo podría ser relevante. 


\section{Conclusión}

Nuestros resultados sugieren que los haplotipos rs11568818(A)-rs11225395(T)-rs2276109(A)rs2252070(A) y rs11568818(A)-rs11225395(C)rs2276109(A)-rs2252070(G) confieren un incremento del riesgo de CCR, mientras que los haplotipos rs11568818(G)-rs11225395(C)-rs2276109(A)-rs2252070(A) y rs11568818(A)-rs11225395(T)-rs2276109(A)rs2252070(G) actúan como un factor protector para CCR. Estuvo presente DL entre rs2276109 (MMP12) y rs2252070 (MMP13). Además, sería importante realizar más estudios para mostrar el posible impacto que un único alelo podría tener como parte de un haplotipo. Por otro lado, las SNV rs11225395 (MMP8) y rs2276109 (MMP12) no mostraron asociación significativa con CCR. Respecto a rs11225395 (MMP8), este representa el primer reporte en pacientes con CCR.

\section{Agradecimientos}

Ruth Ramírez-Ramírez, Anahí González-Mercado, Miriam Partida Pérez, Alexis Sayuri Suárez-Villanueva y José Miguel Moreno-Ortiz agradecen haber recibido beca CONACyT durante el Doctorado en Genética Humana.

\section{Financiamiento}

Los autores recibieron financiamiento del programa PROSNI de Universidad de Guadalajara.

\section{Conflicto de intereses}

Los autores declaran no tener ningún conflicto de intereses.

\section{Responsabilidades éticas}

Protección de sujetos humanos y animales. Los autores declaran que los procedimientos seguidos están de acuerdo con las regulaciones del comité de ética de investigación clínica relevante y con las del Código de Ética de la Asociación Médica Mundial (Declaración de Helsinki).

Confidencialidad de los datos. Los autores declaran haber seguido los protocolos de su centro de trabajo sobre la publicación de datos de pacientes.
Derecho a la privacidad y consentimiento informado. Los autores han obtenido el consentimiento informado por escrito de los pacientes o sujetos mencionados en el artículo. El autor correspondiente está en posesión de este documento.

\section{Bibliografía}

1. Douaiher J, Ravipati A, Grams B, Chowdhury S, Alatise O, Are C. Colorectal cancer-global burden, trends, and geographical variations. J Surg Oncol. 2017;115:619-30.

2. Bray F, Ferlay J, Soerjomataram I, Siegel RL, Torre LA, Jemal A. Global cancer statistics 2018: GLOBOCAN estimates of incidence and mortality worldwide for 36 cancers in 185 countries. CA Cancer J Clin. 2018:683:94-424.

3. Mathonnet M, Perraud A, Christou N, Akil H, Melin C, Battu S, et al. Hallmarks in colorectal cancer: angiogenesis and cancer stem-like cells. World J Gastroenterol. 2014;20:4189-96.

4. Cui N, Hu M, Khalil RA. Biochemical and biological attributes of matrix metalloproteinases. Prog Mol Biol Transl Sci. 2017;147:1-73.

5. Falk $\mathrm{P}$, Jonsson A, Swartling $T$, Asplund D, Ivarsson ML. Role of matrix metalloproteinases in tumour invasion: immunohistochemistry of peritoneum from peritoneal carcinomatosis. Med Oncol. 2018;35:64.

6. Woo M, Park K, Nam J, Kim JC. Clinical implications of matrix metalloproteinase-1, $-3,-7,-9,-12$, and plasminogen activator inhibitor-1 gene polymorphisms in colorectal cancer. J Gastroenterol Hepatol. 2007;22:1064-70.

7. Yueh T, Wu CN, Hung YW, Chang WS, Fu CK, Pei JS, et al. The contribution of MMP-7 genotypes to colorectal cancer susceptibility in Taiwan. Cancer Genomics Proteomics. 2018;15:207-12.

8. Van Nguyen S, Skarstedt M, Löfgren S, Zar N, Anderson RE, Lindh M, et al. Gene polymorphism of matrix metalloproteinase-12 and -13 and association with colorectal cancer in Swedish patients. Anticancer Res. 2013:33:3247-50.

9. Gustincich S, Manfioletti G, Del Sal G, Schneider C, Carninci P. A fast method for high-quality genomic DNA extraction from whole human blood. Biotechniques. 1991;11:298-302.

10. Moreno-Ortiz JM, Gutiérrez-Angulo M, Partida-Pérez M, Peregrina-Sandoval J, Ramírez-Ramírez R, Muñiz-Mendoza R, et al. Association of MMP7-181A/G and MMP13-77A/G polymorphisms with colorectal cancer in a Mexican population. Genet Mol Res. 2014;13:3537-44.

11. Li Y, Jia JH, Kang S, Zhang XJ, Zhao J, Wang N, et al. The functional polymorphisms on promoter region of matrix metalloproteinase-12, -13 genes may alter the risk of epithelial ovarian carcinoma in Chinese. Int Jynecol Cancer. 2009;19:129-33.

12. Excoffier L, Lischer HEL. Arlequin suite ver 3.5: Anew series of programs to perform population genetics analyses under Linux and Windows. Mol Ecol Resour. 2010;10:564-7.

13. Lewentin RC. The interaction of selection and linkage. I. General consideration; heterotic model. Genetics. 1964;49:49-67.

14. Ardlie KG, Kruglyak L, Seielstad M. Patterns of linkage disequilibrium in the human genome. Nat Rev Genet. 2002;3:299-309.

15. Chen SS, Song J, Tu XY, Zhao JH, Ye XQ. The association between MMP12-82A/G polymorphism and susceptibility to various malignant tumors: a meta-analysis. Int J Clin Exp Med. 2015;8:10845-54

16. Kader AK, Shao L, Dinney CP, Schabath MB, Wang Y, Liu J, et al. Matrix metalloproteinase polymorphisms and bladder cancer risk. Cancer Res. 2006;66:11644-8.

17. Yoon S, Kuivaniemi H, Gatalica Z, Olson JM, Butticè G, Ye S, et al. MMP13 promoter polymorphism is associated with atherosclerosis in the abdominal aorta of young black males. Matrix Biol. 2002;21:487-98.

18. Vairaktaris E, Yapijakis C, Nkenke E, Serefoglou ZC, Chatzitheofylaktou A, Vassiliou S, et al. A metalloproteinase-13 polymorphism affecting its gene expression is associated with advanced stages of oral cancer. Anticancer Res. 2007;27:4027-30.

19. Jormsjo S, Ye S, Moritz J, Walter DH, Dimmeler S, Zeiher AM, et al. Allele-specific regulation of matrix metalloproteinase-12 gene activity is associated with coronary artery luminal dimensions in diabetic patients with manifest coronary artery disease. Circ Res. 2000;86:998-1003.

20. Hung YW, Tsai CW, Wu CN, Shih LC, Chen YY, Liu YF, et al. The contribution of matrix metalloproteinase-8 promoter polymorphism to oral cancer susceptibility. In Vivo. 2017;31:585-90.

21. Pei JS, Chang WS, Hsu PC, Hung YW, Cheng SP, Tsai CW, et al. The contribution of MMP-8 promoter genotypes to childhood leukemia. In Vivo. 2017;31:1059-64.

22. Park HS, Ko KH, Kim JO, An HJ, Kim YR, Kim JH, et al. Association study between the polymorphisms of matrix metalloproteinase (MMP) genes and idiopathic recurrent pregnancy loss. Genes (Basel). 2019;10:347. 